\title{
Recorrido por la Realidad de los Laboratorios de Anatomía Humana de 12 Universidades Chilenas
}

\author{
Journey Through the Reality of Human Anatomy Laboratories of 12 Chilean Universities
}

\author{
Gonzalo Tiznado-Matzner'; Sandra Bucarey-Arriagada ${ }^{1} \&$ Pedro Christian Aravena $^{1,2}$
}

TIZNADO-MATZNER, G.; BUCAREY-ARRIAGADA, S. \& ARAVENA, P. C. Recorrido por la realidad de los laboratorios de anatomía humana de 12 universidades chilenas. Int. J. Morphol., 37(1):17-21, 2019.

RESUMEN: La enseñanza de la anatomía humana ha estado ligada históricamente al estudio de cadáveres humanos mediante la observación y la disección. A pesar del paso de los años y los avances en los textos e instrumentos multimediales, no se concibe su educación, sin incorporar los procesos de enseñanza-aprendizaje que se llevan a cabo en los laboratorios de anatomía. El propósito de esta investigación fue describir los recursos físicos, educativos, humanos y los métodos de fijación y conservación de las muestras cadavéricas de 12 universidades chilenas. Se desarrolló un estudio descriptivo donde se visitaron 12 laboratorios de anatomía humana, de los cuales 10 fueron remodelados posterior al año 2000. La capacidad máxima que presentaron fue de 103 estudiantes en promedio. Todos refirieron emplear algún tipo de material cadavérico, pero sólo 3 dijeron poseer un stock que les permite realizar prácticos íntegramente con estos elementos. Para la fijación y conservación el $75 \%$ utiliza una fórmula con bajo porcentaje de formaldehído y otro $17 \%$ lo utiliza a gran concentración. El promedio de los docentes que participan en los laboratorios fue de 11,7 por universidad, de los cuales sólo un 14,2\% presentan postítulo o postgrado en la especialidad. Se aprecia una escasa homogeneidad en la implementación de recurso humano, material cadavérico y métodos de conservación libre de formalina entre los laboratorios de anatomía humana de la universidades chilenas. Se requiere intercambiar experiencias, estandarizar y reconocer las mejores prácticas.

PALABRAS CLAVE: Laboratorios; Anatomía; Universidades Chilenas.

\section{INTRODUCCIÓN}

La anatomía es una asignatura obligatoria en la mayoría de las carreras de la salud, cuyo proceso se inicia a partir de textos clásicos, en los cuales se privilegia la descripción detallada de cada estructura del cuerpo humano. Actualmente existen autores (Moore et al., 2013; Pró, 2014; Drake et al. 2015) que brindan un conocimiento cada vez más didáctico para facilitar su comprensión. Sin embargo, a pesar de los años y los avances en los textos e instrumentos multimediales, no se concibe la enseñanza de esta disciplina sin incorporar los procesos de enseñanza-aprendizaje en los laboratorios de anatomía, lugar donde se realizan las actividades prácticas a través de la utilización de cadáveres, modelos anatómicos, softwares y otros elementos, donde los alumnos tienen la oportunidad de ver y tocar las estructuras aprendidas en forma tridimensional, lo que favorece una mejor internalización de los conceptos aprendidos. Sin ir más lejos, autores como Inzunza \& Bravo (1999) y García-Hernández (2003), afirman que el práctico de anatomía es único, inelu- dible, irreemplazable, irrecuperable e indispensable para realizar una adecuada comprensión de la anatomía.

A nivel internacional, existe consenso que hay que buscar un trabajo colaborativo, que permita optimizar la utilización de los laboratorios. Estos deberían involucrar la incorporación de nuevas metodologías de aprendizaje, asociado al uso de tecnologías que permitan complementar el estudio del cadáver (Benninger et al., 2014). Otro enfoque, bien descrito por la literatura, es el énfasis en solucionar el tema de la exposición al formaldehido, dado que es el fijador y conservador más usado en el material cadavérico.

Referente al estado de los laboratorios de anatomía de las universidades chilenas, la información es inexistente, al igual que la falta de normativas claras que pudieran permitir una homologación y fijación de estándares nacionales. Esto hace necesario comprender procedimientos, tec-

\footnotetext{
${ }^{1}$ Instituto de Anatomía, Histología y Patología, Facultad de Medicina. Universidad Austral de Chile, Chile.

${ }^{2}$ Escuela de Odontología. Facultad de Medicina, Universidad Austral de Chile, Chile.

Financiamiento: Esta investigación contó con el financiamiento de la Dirección de Investigación y Desarrollo de la Universidad Austral de Chile, en el año 2015 ( $\mathrm{N}^{\mathrm{o}}$ del proyecto S-2015-79).
} 
nologías y metodologías de trabajo, lo que llevaría a visualizar las líneas de desarrollo y conocimientos, que permitirían una proyección eficiente y colaborativa, logrando impactar positivamente en los laboratorios del país, tanto en la modernización de éstos como en su funcionamiento.

Es por tal motivo que esta investigación se basa en describir los recursos de infraestructura, técnicos, físicos, humanos y educativos con los que disponen los laboratorios de Anatomía Humana de las universidades chilenas.

\section{MATERIAL Y MÉTODO}

Se realizó un estudio descriptivo basado en entrevistas y visitas a los laboratorios de Anatomía Humana de 12 Universidades Chilenas entre los años 2015 y 2016. Para el registro de datos, se diseñó un instrumento basado en registro de locales y del personal que participó en la entrevista y de cuatro aspectos de los laboratorios de anatomía humana: Recursos físicos (año de construcción, metros cuadrados de construcción, capacidad de estudiantes, número y tipo de material de mesa de trabajo, tipo de iluminación, tipo de ventilación), recursos educativos (Tipo de material de estudio tales como material cadavérico o modelos anatómicos, uso de softwares, origen del material cadavérico); Uso de método de fijación y conservación de muestras cadavéricas (material de fijación, de conservación y conocimiento de norma vigente sobre su uso) y tipo de recurso humano (número de docentes, número de técnicos y especialización de docentes y técnicos).

Para analizar la fachada del instrumento, fue enviado vía correo electrónico a 10 integrantes de la Sociedad Chilena de Anatomía quienes sugirieron corregir el formato de preguntas (realizar preguntas abiertas) y agregar datos respecto a número de docentes con postgrado y experiencia en disección y conservación de cadáveres.

Las universidades se seleccionaron de acuerdo a los antecedentes aportados por la Sociedad Chilena de Anatomía en su página Web (http:// www.sociedadchilenadeanatomia.cl). Del total de escuelas o laboratorios en Chile doce de ellos accedieron a participar voluntariamente del estudio:

- Universidad de Tarapacá.

- Universidad de Antofagasta.

- Pontificia Universidad Católica de Valparaíso.

- Universidad de Playa Ancha.

- Universidad de Chile.

- Pontificia Universidad Católica de Chile.

- Universidad Diego Portales.
- Universidad de Talca.

- Universidad Católica del Maule.

- Universidad de Concepción.

- Universidad de La Frontera.

- Universidad de Magallanes.

Las visitas a las universidades se realizaron entre Septiembre del 2015 a noviembre del 2016, previa coordinación con el profesor o encargado del laboratorio de anatomía humana. En ello, el investigador principal entrevistó al personal académico y técnico, registrando los datos en una ficha diseñada para ello. En caso de preguntas abiertas, las entrevistas fueron grabadas y digitalizadas, para luego obtener información concreta respecto a las los aspectos relevantes de esta investigación.

Todos los datos fueron tabulados en una base de datos electrónica (Google Drive, Google Inc. Mountain View, CA, USA) y analizados mediante estadística descriptiva. Se presentan los resultados en valores de frecuencia y figuras de gráficos.

\section{RESULTADOS}

A) Recurso Físico. De los 12 laboratorios visitados, 10 fueron remodelados en el siglo actual, de los restantes: uno fue remodelado a principios del siglo XX y el otro fue construido a finales del siglo XX, al cual no se le han realizado arreglos substanciales. Al ser consultados por el área de construcción, no se pudo obtener datos fidedignos. Sin embargo, se pudo establecer que la capacidad máxima era de 103 estudiantes en promedio, entretanto que el promedio de mesas de trabajo por laboratorio fue de 14 (Fig. 1). Respecto al material de las

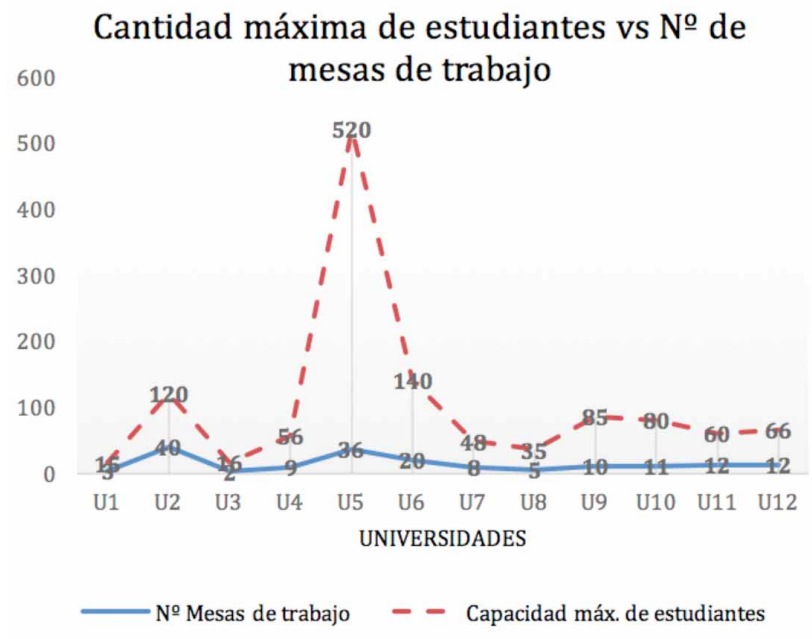

Fig. 1. Comparativa entre capacidad máxima de estudiantes por cantidad de mesas de trabajo. 
mesas, 10 laboratorios utilizaban mesas de acero inoxidable, de ellos, 3 ademas contaban con mesas de melamina y 2 con mesas de mármol. Un laboratorio contaba únicamente con mesas de mármol y otra con mesas de grafito.

En relación a la iluminación se aprecia que 9 laboratorios presentaban una mezcla de luz artificial con luz natural proveniente de las ventanas (Fig. 2), mientras que el sistema más común de ventilación fue la utilización de extractor de aire en combinación con ventilación natural a través de la apertura de ventanas. Sólo 3 universidades presentaban equipamiento de inyección y extracción de aire (Fig. 2). No obstante, a pesar de tener equipamiento para la ventilación ninguna refiere haber realizado estudios de medición sobre calidad del aire, presencia de partículas contaminantes y cálculo de renovación de aire por hora.

\section{Ventilación/Iluminación}

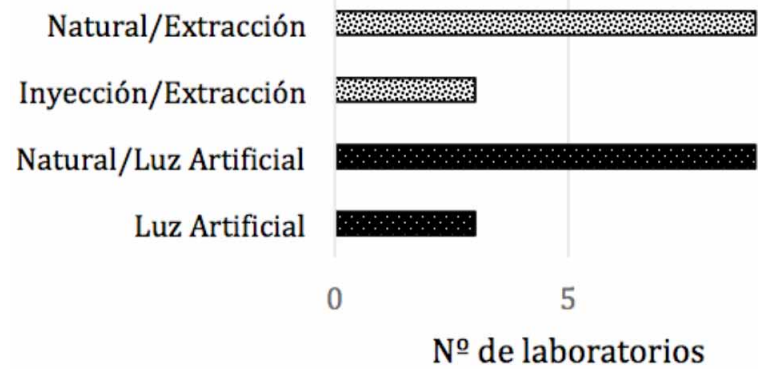

Fig. 2 Tipos de ventilación e iluminación observada en 12 laboratorios de anatomía en Chile.

B) Recursos educativos. Todas las universidades relataron disponer de material cadavérico para la enseñanza, pero sólo 3 universidades poseen un stock que les permite realizar los pasos prácticos, íntegramente en torno a éste. En tanto que los demás laboratorios organizan sus prácticos, complementando el material cadavérico disponible con modelos anatómicos y en menor medida con algún tipo de software de anatomía. En sólo un laboratorio el recurso principal fue la utilización de un software interactivo de anatomía. Frente a la pregunta de dónde obtienen el material cadavérico, sólo 3 indicaron que a través de protocolos de donación de cadáveres. Las otras 9 universidades poseen algún tipo de protocolo, pero refieren que no funciona correctamente, debido a escasos donantes u organización deficiente, por ende el material que poseen, cuya procedencia en muchos casos es desconocida, debido a su antigüedad, es escaso y añejo. Frente a esta problemática una de las soluciones utilizadas por 4 establecimientos, fue la adquisición de material cadavérico en el extranjero, en forma de piezas plastinadas o cadáveres fijados.
C) Utilización de métodos de fijación y conservación de muestras cadavéricas. De los entrevistados 9 refirieron la utilización de una fórmula compuesta por cloruro de sodio, nitrato de sodio, glicerina, alcohol metílico, cloruro de benzalconio, esencia de eucalipto y una pequeña concentración de formaldehído para la fijación y la conservación de muestras cadavéricas, 2 utilizan principalmente formaldehído a diferentes concentraciones y en 1 caso no sabe concretamente cual es la sustancia, debido que el material se encontraba antes de que asumiera su cargo.

Frente a la pregunta si conoce la normativa de exposición al formaldehído, descrito en el decreto supremo 594, artículo 61 del Ministerio de Salud de Chile, todos los entrevistados afirmaron estar al tanto, no obstante, sólo 2 universidades afirmaron realizar mediciones periódicas de la concentración de gases tóxicos en los laboratorios, 4 haberlas realizado alguna vez y 7 no tener conocimiento de este procedimiento. A pesar de esto, ninguna entidad ha certificado alguna enfermedad producto de la exposición a gases nocivos como el formaldehído.

D) Recurso humano. La cantidad de docentes de anatomía humana en cada universidad es muy variable (Fig. 3), debido principalmente a la cantidad de cupos por carrera que define cada establecimiento. No obstante, se obtuvo un promedio de 11,7 (Mín. 4; Máx. 28) docentes por universidad, de los cuales el 3,9 (Mín. 1; Máx. 7), que representa el 30,7 $\%$, están contratados, en tanto que los restantes se encuentra a honorarios. Además, se obtuvo que sólo el 14,2 \% presentaba un postítulo o postgrado en morfología (Fig. 3).

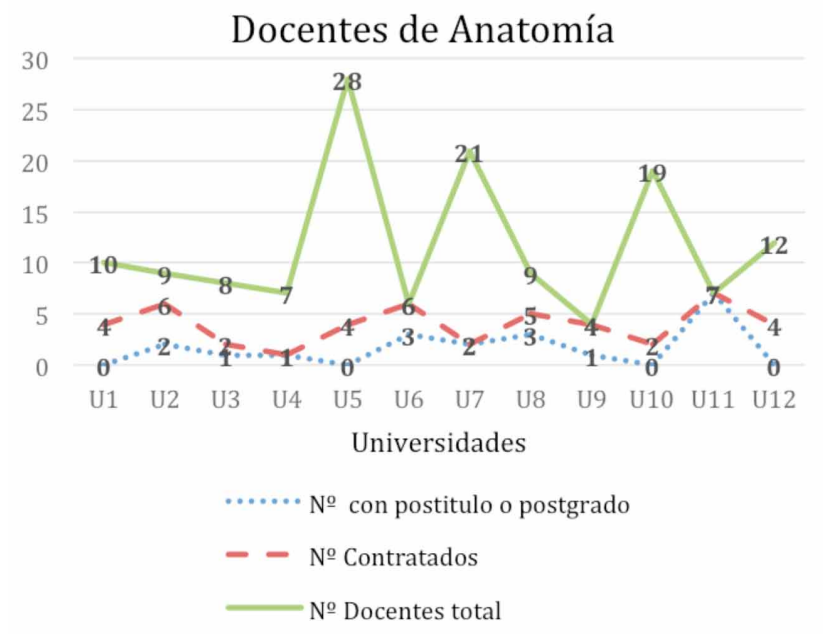

Fig. 3. Comparativa entre cantidad de docentes vs docentes contratados vs docentes con postítulo o postgrado en morfología.

Por el lado de los técnicos de laboratorio, 10 universidades cuentan con al menos uno, en tanto que los 2 restantes carecían de éstos. Las funciones más comunes a desa- 
rrollar son las de ser encargados de fijar y conservar las muestras, preparación de éstas para los pasos prácticos y organización de los laboratorios. Sólo en 4 universidades los técnicos tenían estudios relacionados con la fijación y conservación de muestras.

\section{DISCUSIÓN}

En el recorrido realizado por los laboratorios de anatomía humana de 12 universidades chilenas, entre los años 2015 y 2016, se ha demostrado la gran variabilidad existente en el tipo de construcción e implementación de éstos. Sin embargo, la mayoría comparte la característica de ser edificaciones modernas, que han sido remodelados a principios del siglo XXI. Esto les permite asemejarse a laboratorios internacionales que han abandonado los antiguos salones de disección, que consistían en un mesón central rodeado de sillas en forma de anfiteatro, en el cual el profesor se ubicaba junto al cadáver en el centro y los alumnos en las sillas alrededor como meros espectadores de la clase. Gracias al trabajo conjunto entre arquitectos y anatomistas, los laboratorios han ido evolucionando y se ha preferido la utilizaciones de salones abiertos con mesones de acero inoxidable, en los cuales grupos de alumnos pueden trabajar con muestras anatómicas, permitiéndoles observarlas y manipularlas a la vez. La sala de disección y almacenaje, por lo general, se ubica en una sala aparte (Trelease, 2006). Lo que respecta a nuestra realidad, se observa la utilización de mesones de melamina y mármol, pero mayoritariamente de acero inoxidable. Caso especial es la utilización de mesones de grafito, que datan del siglo pasado.

Otro ámbito importante en los laboratorio es el uso de formaldehído para la fijación y conservación del material cadavérico debido a su bajo costo y su gran eficiencia. Sin embargo, presenta gran volatilidad y es considerada un sustancia altamente tóxica (Ohmichi et al., 2006). Debido a esto un laboratorio de anatomía debe presentar un sistema de inyección de aire y extractores que permitan una renovación constante del flujo de aire, donde la extracción debe ser guiada hacia el suelo, para evitar su inhalación (Goldman, 2010). En la mayoría de los laboratorios los controles de presencia de gases tóxicos son escasos y los cálculos de tiempos de exposición prácticamente inexistentes, como sugiere Mirabelli et al. (2011). Desde esta perspectiva, Kunugita et al. (2004) y Yamato et al. (2005) efectuaron mediciones de concentración de formaldehído, evidenciando la alta concentración de este químico en el ambiente de los laboratorios. Frente a esto implementaron mesones con sistemas de extracción localizada, reportando una disminución significativa de la presencia de esta sustancia. Como pudimos observar, en Chile la utilización exclusiva de formaldehído para la fijación y conserva- ción de material cadavérico está siendo reemplazado por una fórmula compuesta por cloruro de sodio, nitrato de sodio, glicerina, alcohol metílico, cloruro de benzalconio, esencia de eucalipto y una pequeña concentración de formaldehído. No obstante no se ha logrado reemplazar completamente el empleo de éste último, por este motivo llama la atención que todas las personas entrevistadas afirmen tener conocimiento del decreto supremo $N^{\circ}$ 594, artículo 61 del Ministerio de Salud de Chile (Ministerio de Salud, 2000) que modifica el formaldehído de una sustancia A.2. sospechosa de ser cancerígeno a A.1. comprobadamente cancerígeno. Sin embargo, a pesar de esto, la mayoría relata que no se realizan mediciones de gases tóxicos periódicamente, sumado a que el sistema de ventilación comúnmente utilizado es el de extractor de aire en las paredes, dejando la inyección de aire a la apertura de ventanas, lo que imposibilita tener conocimiento exacto de la renovación de aire por hora. Para combatir esto, Ohmichi et al. (2007), experimentaron con mesones equipados con un dispositivo fotocatalítico, el cual fuerza el paso del formaldehído a través de filtros cubiertos de óxido de titanio, expuesto a rayos ultravioleta y carbón activado, descomponiéndolo y atrapando sus elementos nocivos. Mediante este sistema lograron disminuir entre un 70 y $80 \%$ la concentración de este gas. Para evitar estos gastos y contratiempos en la construcción o renovación de laboratorios, las universidades han decidido ir reemplazando todo material cadavérico con modelos anatómicos y softwares con tecnología digital (Wessels et al., 2015). Algo no tan lejano a nuestra realidad donde sólo 3 universidades fueron capaces de mencionar que poseen gran volumen de material cadavérico, obtenido fundamentalmente por un sistema de donaciones bien organizado, mientras que las demás instituciones sólo poseen material óseo o mantienen piezas deterioradas, que fueron donados hace más de 20 años y que no han ido siendo renovadas con el pasar de los años, como consecuencia de protocolos de donaciones ineficientes. Esto podría impactar en la enseñanza de la anatomía, como sugieren Pérez et al. (2007), quienes afirman que los prácticos de anatomía constituyen el primer contacto del alumno con la muerte, desarrollando mecanismos de adaptación en su futuro profesional, además de ser un elemento motivador como manifiestan Collipal \& Silva (2011). En este mismo sentido, Babinski et al. (2003) mostraron que el estudio práctico en el cadáver, fue considerado por el $80 \%$ de los alumnos como muy importante, aumentando a un $85,1 \%$ cuando se les pregunta sobre la influencia en el aprendizaje tras la utilización de cadáveres en los pasos prácticos.

Otro de los problemas que pudimos pesquisar, es que si bien las instituciones presentan un número significativo de docentes que realizan clases de anatomía, es pequeño el porcentaje de éstos con estudios formales de postítulo o postgrado en el área de la morfología, algo que ya fue reportado por Guiraldes et al. (2001) quienes indicaron que uno de los ma- 
yores inconvenientes que presenta la enseñanza de esta disciplina, junto a la cada vez mayor escasez de cadáveres, es la improvisación del cuerpo docente. Similar situación ocurre con los técnicos de laboratorio, quienes en muchos casos son los encargados de fijar y conservar las muestras, para lo cual el conocimiento de anatomía y química, es esencial en su desarrollo laboral

El presente estudio muestra como los laboratorios se han adaptado a la conformación de laboratorios modernos, donde la mayoría trabaja con material cadavérico, cada vez más escaso, pero bien aprovechado, los que además son complementados con modelos anatómicos y softwares. No obstante, la utilización de material cadavérico conlleva la presencia de sustancias nocivas para la salud, que hasta el momento no ha sido solucionado, exponiendo tanto a docentes como alumnos a su efecto perjudicial.

Como conclusión, en este trabajo se presenta las condiciones físicas, estructurales, materiales y humanas de los laboratorios de anatomía humana de 12 universidades chilenas. Se sugieren nuevos estudios aumentando el número de universidades y crear protocolos o guías de creación y mejoramiento de laboratorios de anatomía, favoreciendo las condiciones en las que se desarrolla el ambiente educacional.

TIZNADO-MATZNER, G.; BUCAREY-ARRIAGADA, S. \& ARAVENA, P. C. Journey through the reality of human anatomy laboratories of 12 Chilean Universities. Int. J. Morphol., 37(1):17-21, 2019.

SUMMARY: The teaching of human anatomy has historically been linked to the study of human corpses through observation and dissection. Despite the advances in multimedia texts and instruments over the years, anatomy education is not understood without incorporating the teaching-learning processes in the anatomy laboratories. The purpose of thisresearch was to describe the physical, educational, human resources and methods of fixation and conservation of cadaveric samples from 12 Chilean universities. A descriptive study was carried out where 12 human anatomy laboratories were visited, of which 10 were remodeled after the year 2000 . The maximum capacity average of the laboratories was 103 students. All those interviewed reported using some type of cadaveric material, but only 3 of the labs have sufficient stock to allow these elements to be used in real practice. For fixation and preservation, $75 \%$ of labs use a low percentage formaldehyde, while $17 \%$ use a high concentration. The average of teachers that participate in the laboratories was of 11.7 per university, of which only $14.2 \%$ present graduate or postgraduate degree in the specialty. Homogeneity is scarce in Chilean universities in reference to the implementation of human resources, cadaveric samples and formalin-free conservation methods in human anatomy laboratories. Therefore, it is important to exchange experiences, standardize and recognize the best practices in this field.

KEY WORDS: Laboratories; Anatomy; Chilean Universities.

\section{REFERENCIAS BIBLIOGRÁFICAS}

Babinski, M. A.; Sgrott, E. A.; Luz, H. P.; Brasil, F. B.; Chagas, M. A. \& AbiduFigueiredo, M. The relationship of the students with corpse in the practical study of anatomy: the reaction and influence in the learning. Int. J. Morphol., 21(2):137-42, 2003.

Benninger, B.; Matsler, N. \& Delamarter, T. Classic versus millennial medical lab anatomy. Clin. Anat., 27(7):988-93, 2014.

Collipal, L. E. \& Silva, M. H. Study of anatomy in cadavers and anatomical models. Impression of students. Int. J. Morphol., 29(4):1181-5, 2011

Drake, R.; Wayne, A. \& Mitchel, A. Gray. Anatomía para Estudiantes. $3^{\mathrm{a}} \mathrm{ed}$ Barcelona, Elsevier, 2015.

García-Hernández, F. Evaluation of the practical learning of the human anatomy for dentistry in the University of Antofagasta, Chile. Int. J. Morphol., 21(1):43-7, 2003.

Goldman, E. Building a low-cost gross anatomy laboratory: a big step for a small university. Anat. Sci. Educ., 3(4):195-201, 2010.

Guiraldes, H.; Oddó, H.; Mena, B.; Velasco. N. \& Paulos, J. Teaching human anatomy: experience and challenge in a medical school. Rev. Chil. Anat., 19(2):205-12, 2001

Inzunza, O. \& Bravo, H. Impact of two software of human anatomy in the performance of the students practical knowledge. Rev. Chil. Anat., 17(2):205-9, 1999.

Kunugita, N; Arashidani, K; Yamato, H; Tanaka, I; Nakashima, Y \& Kikuta, A. Evaluation of formaldehyde exposure during a gross anatomy dissection course. J. Univ. Occup. Environ. Health, 26:337-48, 2004.

Ministerio de Salud. Decreto 594. Santiago de Chile, Biblioteca Nacional de Chile, 2000. Disponible en: http://www.achs.cl/portal/trabajadores/ Documents/ds-594.pdf

Mirabelli, M. C.; Holt, S. M. \& Cope, J. M. Anatomy laboratory instruction and occupational exposure to formaldehyde. Occup. Environ. Med., 68(5):375-8, 2011.

Moore, K.; Dailey, A. \& Agur, A. Anatomía con Orientación Clínica. $7^{\mathrm{a}}$ ed. Barcelona, Lippincott Williams \& Wilkins, 2013.

Ohmichi, K.; Komiyama, M.; Matsuno, Y.; Sawabe, Y.; Miyaso, H.; Fukata, H.; Ohmichi, M.; Kadota, T.; Nomura, F. \& Mori, C. Relationship between exposure to formaldehyde and immunoglubulin $\mathrm{E}$ (IgE) production during the gross anatomy laboratory. J. Health Sci., 52(6):642-7, 2006.

Ohmichi, K.; Matsuno, Y.; Miyaso, H.; Yamamoto, H.; Toriuchi, M.; Shimane, M. \& Mori, C. Pilot study of a dissection table for gross anatomy laboratory equipped with a photocatalytic device that decomposes formaldehyde. $J$. Occup. Health, 49(6):499-503, 2007.

Pérez, M. M.; Riba, N. P.; Ortiz Sagristà, J. C.; Martinez, A. \& Götznes García V. Anatomía Humana: estudio de las reacciones de los estudiantes de primero de medicina ante la sala de disección. Educ. Med., 10(2):105-13, 2007.

Pró, E. A. Anatomía Clínica. 2ª ed. Buenos Aires, Médica Panamericana, 2014.

Trelease, R. B. Anatomy meets architecture: designing new laboratories for new anatomists. Anat. Rec. B New Anat., 289(6):241-51, 2006.

Wessels, Q.; Vorster, W. \& Jacobson, C. Designing Gross Anatomy Laboratory to Meet the Needs of Today's Leaner. In: Chan, L. \& Pawlina, W. (Eds.). Teaching Anatomy. Cham, Springer, 2015.

Yamato, H.; Nakashima, T.; Kikuta, A.; Kunugita, N.; Arashidani, K.; Nagafuchi, Y. \& Tanaka, I. A novel local ventilation system to reduce the levels of formaldehyde exposure during a gross anatomy dissection course and its evaluation using real-time monitoring. J. Occup. Health, 47(5):450-3, 2005.

\section{Dirección para correspondencia: \\ Gonzalo Tiznado Matzner \\ Universidad Austral de Chile \\ Campus Isla Teja S/N \\ Valdivia \\ CHILE}

Recibido : 13-07-2018

Aceptado: 16-10-2018

Email: gonzalo.tiznado@uach.cl 